\title{
Multisystem Inflammatory Syndrome in Children Temporarily Associated with SARS-CoV-2
}

\author{
Vinod H. Ratageri ${ }^{1}$ (D) Gayatri R. Pawar ${ }^{1} \cdot$ Guruprasad N $^{1} \cdot$ Shadab B. Maldar ${ }^{1} \cdot$ Shivanand Illau ${ }^{1}$
}

Received: 6 December 2020 / Accepted: 3 February 2021/ Published online: 24 January 2021

(C) Dr. K C Chaudhuri Foundation 2021

To the Editor: COVID-19 in children is largely benign, however, multisystem inflammatory syndrome in children (MISC) associated with SARS-CoV-2 can lead to serious and lifethreatening illness in previously healthy children [1]. In this prospective observational series, we describe clinical profile and outcome of children with MIS-C.

A total of 8 children met WHO criteria [2] for MIS-C during September and October 2020. The male to female ratio was $3: 1$ with median age of 4.65 y [interquartile range (IQR) 3.1-7.2 y]. All children were healthy before this illness. All children had fever with mean duration of $6 \pm 1.72 \mathrm{~d}$. The common systems of clinical presentation were cardiovascular 7 $(87.5 \%)$, gastrointestinal $4(50 \%)$, and respiratory $2(25 \%)$. The other manifestations include shock 5 (67.5\%), hypotension 4 (50\%), hypoxemia 4 (50\%), and generalized erythematous rash $4(50 \%)$.

Serological evidence of SARS-CoV-2 infection was present in 7 (87.5\%), which also includes 1 RT-PCR-positive child. One child had exposure to COVID-19. All children were investigated for dengue, rickettsial fever, chikungunya, and sepsis and were found negative. Thrombocytopenia was seen in 7 (87.5\%) children; leucopenia and lymphopenia in 1 child each. The inflammatory markers were elevated in all the children. Two children had liver dysfunction and none had abnormal renal functions.

Five $(67.5 \%)$ had abnormal 2D ECHO, of which, 3 had severe left ventricular dysfunction. None of them had coronary artery abnormalities during the hospital stay. Fifty percent (4) of children had hypoxemia needing oxygen support by noninvasive ventilation. Inotrope/vasopressor support was needed in 4 children $(50 \%)$. All the 8 children were treated

Vinod H. Ratageri

ratageri@ rediffmail.com

1 Department of Pediatrics, Karnataka Institute of Medical Sciences, Hubli, Karnataka 580021, India with intravenous immunoglobulin (IVIG) $(2 \mathrm{~g} / \mathrm{kg})$. In addition, 6 children received methyl prednisolone $(10-30 \mathrm{mg} / \mathrm{kg})$. No mortality observed.

The striking cardiac manifestations in our series were cardiogenic shock and abnormal echocardiography in the form of left ventricular (LV) dysfunction. The mechanism underlying myocardial dysfunction in MIS-C has not been yet fully elucidated. Possible causes as seen in adults with COVID-19 include acute myocarditis, hypoxic injury, and ischemic injury caused by cardiac microvascular damage or coronary artery disease $[3,4]$.

\section{Declarations}

Conflict of Interest None.

\section{References}

1. Dufort EM, Koumans EH, Chow EJ, et al. Multisystem inflammatory syndrome in children in New York state. N Engl J Med. 2020;383:347-58.

2. World Health Organization. Multisystem inflammatory syndrome in children and adolescents with COVID-19. Available at: https://www. who.int/publications/i/item/multisystem-inflammatory-syndromein-childrenand-adolescents-with-covid-19. Accessed 28 Nov 2020.

3. Belhadjer Z, Méot M, Bajolle F, et al. Acute heart failure in multisystem inflammatory syndrome in children (MIS-C) in the context of global SARS-CoV-2 pandemic. Circulation. 2020;142:429-36.

4. Madjid M, Safavi-Naeini P, Solomon SD, Vardeny O. Potential effects of coronaviruses on the cardiovascular system: a review. JAMA Cardiol. 2020;5:831-40.

Publisher's Note Springer Nature remains neutral with regard to jurisdictional claims in published maps and institutional affiliations. 